\title{
Estudo de calibração do Questionário de Frequência Alimentar para Adolescentes (QFAA)
}

\author{
Calibration Study of the Food Frequency Questionnaire \\ for Adolescents (AFFQ)
}

Silvia M aria Voci ${ }^{1}$

Betzabeth Slater ${ }^{1}$

Marina Vieira da Silva ${ }^{2}$

Dirce M aria Lobo M archioni ${ }^{1}$

M aria do Rosário Dias de Oliveira Latorre ${ }^{1}$

${ }^{1}$ Departamento deN utrição, Faculdade de SaúdePública, Universidade deSão Paulo. Av. Doutor Arnaldo 715, 20 andar. 01246-904 São Paulo SP. smvoci@usp.br ${ }^{2}$ Departamento de Agroindústria, Alimentose Nutrição, Escola Superior deAgricultura Luiz de Queiroz, Universidade de São Paulo.
Abstract In order to establish calibration factors of the Adolescent Food Frequency Questionnaire (AFFQ), 74 boys and girls from Piracicaba (SP, Brazil) with ages ranging from 10 to 14 took part in the study. Dietary intake (assessed by the food frequency questionnaire and 24-hour recall) was assessed and adjusted for energy intake. Descriptive statistics, variance analysis using one classification factor, Pearson's correlation coefficientsand linear regression were performed. The average of two 24-hour recalls was used as a reference for calibration of data. Calibration coefficients $(\lambda)$ ranged from -0.07 (iron) to 0.40 (vitamin C) re vealing substantial error in the dietary method tested, albeit similar to those observed in theliterature. As these coefficients were low, they indicate the need for reformulating the instrument regarding some nutrients, though application was not considered advisable for correcting information on iron and retinol. The methodology used to calibrate dietary data can consider measurement error in the assessment when its assumptions are respected, sinceviolations of theseassumptionsmay lead to other errors that are difficult to predict. Key words Calibration, Diet, M easurement error, Linear regression
Resumo Para determinar fatores de calibração do Questionário de Frequência Alimentar para Adolescentes (QFAA), participaram do estudo 74 adolescentes de Piracicaba (SP), com idades entre $10 \mathrm{e}$ 14 anos. Dados de consumo alimentar foram levantados por meio de um QFAA e dois recordatórios de 24 horas ajustados pela energi a. Foram realizadas análises descritivas, análise de variância com um fator de classificação, coeficientes de correlação de Pearson e regressão linear. A média dos dois recordatórios foi utilizada como referência para calibrar os dados. Os coeficientes de calibração variaram de -0,07 (ferro) a 0,40 (vitamina C), mostrando substancial erro no método de inquérito dietético testado, mas sendo semelhantes aos observados na literatura. D evido ao fato de se apresentarem baixos, indicam a necessidade de reformulação do instrumento para alguns nutrientes, sendo desaconselhada sua aplicação para corrigir informaç̧ões de ferro e retinol. A metodologia utilizada é capaz de considerar nas análises os erros de mensuração quando suas pressuposições são respeitadas, uma vez que violações desses pressupostos podem levar ao surgimento de outros erros de difícil predição.

Palavras-chave Calibração, Dieta, Erro demedida, Regressão linear 
Introdução

M uitos pesquisadores reconhecem que dados dietéticos, principalmente os obtidos por Questionários de Frequência Alimentar (QFA), estão sujeitos a erros de medida, representando uma limitação importante nas associações entre dieta e doença obtidas em estudos epidemiológicos ${ }^{1}$. 0 erro demedida é definido como a diferença entre o consumo habitual observado e o verdadeiro, incorporando tanto a variação da dieta quanto os componentes de erro resultantes do uso do instrumento ${ }^{1}$. Além disso, outrafontedeerro pode ser proveniente da aplicação de um QFA já existente, devendo-se levar em consideração que esse mesmo instrumento deve ser apropriado e validado para a população que se deseja estudar².

Para interpretar as relações observadas em estudos epidemiológicos, é essencial o entendimento da direção e da magnitude do erro de medida introduzido pelo uso do Q FA, bem como da distorção na associação entre dieta e doença3.

Para lidar com erros de medida em variáveis de exposição e para obter uma estimativa de risco corrigida, Rosner et al. ${ }^{4}$ introduziram o método estatístico da regressão de calibração.

Segundo Kynast-Wolf et al. ${ }^{2}$, um dos principais escopos do método de calibração éa eliminação (ou a redução) do viés por meio da nova gradação dos valores, no sentido em que as novas médias serão iguais às obtidas por recordatórios de 24 horas (R24h) ou diários alimentares (DA). Esta nova gradação também move o nível de ingestão individual em direção ao dado de referência.

Uma vez quenão existe o "padrão-ouro" para a avaliação baseada em relatos da ingestão dietética verdadeira dos nutrientes, a abordagem de regressão de calibração requer a aplicação de um instrumento de referência. A inclinação da re gressão linear dos valores de referência $(R)$ contra os valores obtidos pelo questionário de frequência (Q) deveria ser igual ao fator de calibração que seria obtido se fosse utilizado o valor verdadeiro (desconhecido, $\mathrm{T})^{1}$.

$$
E(R \mid Q)=E(T \mid Q)
$$

Nesse sentido, o fator de calibração $\lambda$ é representado como sendo a inclinação da reta de regressão de $R$ contra os valores do $Q F A(Q)^{5}$.

0 esperado para os parâmetros estimados é um intercepto de aproximadamente zero e uma inclinação deaproximadamente 1,0 , indicando que o questionário não está enviesado. Entretanto, a maioria dos estudos mostra inclinações menores do que 1,0. Esse efeito tem sido denominado de flattened slope e pode ser atribuído ao viés dado pelo relato, ao erro no método de referência devido à variação da ingestão alimentar ao longo do período mensurado, e ao erro devido à conversão dos itens alimentares em nutrientes ${ }^{6}$.

Uma vez que o instrumento original sofreu adaptações, o objetivo do presente estudo foi determinar fatores de calibração para a correção dos dados di etéticos obtidos por QFAA, utilizando-seinformações deadolescentes, alunos deuma escola da rede pública de ensino do município de Piracicaba (SP).

\section{Métodos}

Estetrabal ho éum subestudo inserido no estudo de coorte intitulado Consumo dietético e atividade física como determinantes das mudanças do índice demassa corporal deuma coortedeadolescentes matriculados na rede pública de ensino da cidade de Piracicaba, São Paulo, financiado pela Fundação de Amparo à Pesquisa do Estado de São Paulo (Fapesp).

No processo de amostragem do estudo principal (coorte), foram consideradas informações sobre a prevalência de excesso de peso obtidas em estudos anteriores realizados com população semelhanteno país. Considerou-seerro tipo | de $5 \%$ e erro tipo || de $20 \%$ para o cálculo da amostra. A amostragem utilizada foi a de múltiplos estágios, sendo iniciada pela ordenação das escolas de acordo com as regiões do município. Foram sorteadas 12 escolas da rede pública de ensino de Piracicaba (SP), considerando que 11 estavam localizadas em zonas urbanas e uma em zona rural.

De acordo com Cade et al. ${ }^{7}$, para a realização deste subestudo, 0 ideal seria que a amostra fosse representativa da amostra do estudo principal. Das 12 escolas sorteadas na coorte, selecionou-se uma unidade com localização central e, portanto, situada na zona urbana, contendo turmas de 5 a a 8a séries, com características bastanteheterogêneas por acolher alunos de diversas regiões do município. A presente pesquisa foi realizada nessa escola, que foi subtraída da amostra da coorte.

Ingressaram no subestudo, realizado entre agosto e outubro de 2004, 94 adolescentes de ambos os sexos, voluntários. Foram obtidos dados demográficos, antropométricos (peso e estatura), de maturação sexual e de consumo alimentar. 
Para a avaliação do peso corporal, foram utilizadas balanças eletrônicas do tipo plataforma, com capacidade para $150 \mathrm{~kg}$ e sensibilidade de 100 gramas. 0 dado foi aferido em duplicidade, com os adolescentes vestindo roupas leves e descal ços.

Para a coleta dos dados de altura, utilizou-se estadiômetro com escala em milímetros, fixado em suporte de madeira, de modo que foi mantido um ângulo reto entre o piso e a parede. $A$ aferição foi realizada em duplicidade, com os indivíduos posicionados de pés juntos, calcanhares encostados no suporte de madeira, em postura ereta, olhando para a frente, sem flectir ou estender a cabeça, com o ápice da orel ha e o canto externo do olho posicionados em linha paralela à barra do estadiômetro. A leitura foi realizada em centímetros. Foram utilizadas as médias das medidas de peso e estatura para o cálculo do IMC, procedendo-se à classificação do estado nutricional utilizando-se os valores dereferência propostos pela $\mathrm{WHO}^{8}$.

Vale ressaltar que o enfoque principal do trabalho recai sobre as variáveis relativas ao consumo (representado pelos valores do Q FAA e pelos valores médios dos dois R24h). Assim, para avaliar o consumo alimentar dos alunos, foram aplicados dois R24h e um QFAA semiquantitativo, desenvolvido e validado por Slater et al. ${ }^{9}$, após adaptações realizadas. 0 QFAA reformulado incluiu perguntas sobre 94 itens alimentares, com sete opções de frequências de consumo, sobre um período de seis meses pregressos.

Os R24h foram considerados como método dereferência e aplicados com intervalo entre $30 \mathrm{e}$ 45 dias, utilizando-se material fotográfico especificamente desenvolvido para o estudo. 0 segundo R24h foi aplicado juntamente com o QFAA.

As variáveis dietéticas estudadas foram: energia (Kcal), macronutrientes (g), fibra (g), colesterol $(\mathrm{mg})$, cálcio $(\mathrm{mg})$, ferro $(\mathrm{mg})$, vitamina C $(\mathrm{mg})$ eretinol $(\mu \mathrm{g})$, devido ao fato de o QFAA ter sido validado para esses componentes.

A digitação das informações do QFAA foi realizada utilizando-se o software Dietsys ${ }^{10}$, versão 4.01, procedendo-seà dupla digitação para eliminação de eventuais erros. Os R24h das duas etapas foram digitados no programa N utrition Data System ${ }^{11}$. Ambos os softwares são baseados nos dados de composição das tabelas do United StatesDepartment of Agriculture. Aspreparaçõesque não faziam parte do banco de dados de ambos os softwares foram calculadas e inseridas a partir da própria base de dados desses sistemas.

Para as análises, foram adotados como crité rios de exclusão: (1) idade inferior a 10 anos ou superior a 14 anos; (2) o não preenchimento do QFAA ou de um dos dois R24h; (3) valores de consumo diário de energia inferiores a $500 \mathrm{Kcal}$ ou superiores a $6.000 \mathrm{Kcal}^{12}$. A amostra final foi constituída por 74 indivíduos.

Realizaram-se análises descritivas e de frequência para a totalidade das variáveis do estudo. Todas as variáveis dietéticas utilizadas no modelo de calibração apresentaram distribuição normal.

Cabe ressaltar que as variáveis dietéticas foram ajustadas pela energia, de acordo com 0 método dos resíduos, proposto por Willett e Stampfer ${ }^{13}$.

Os coeficientes de correlação de Pearson $r$ foram corrigidos pela variância intrapessoal dos R24h, a partir de análise de variância com um fator de classificação ${ }^{14}$. Segundo Willett ${ }^{14}$, quando apenas a variável dependente é afetada pelo erro aleatório intrapessoal, o coeficiente de regressão não é atenuado, não havendo a necessidade de correção. Portanto, os fatores de calibração não foram corrigidos pela razão de variância.

Utilizou-se o coeficiente decorrel ação de Pearson para avaliar a relação entre as variáveis dietéticas provenientes do QFAA ea média dos R24h, tanto brutas quanto ajustadas pela energia. Para a calibração, aplicou-se modelo de regressão linear simples, levando-se em consideração a mé dia dos dois R24h. A plicou-se a equação

$$
E(R \mid Q)=v+\lambda Q
$$

representando a regressão dos valores médios do R24h nos obtidos por QFAA ${ }^{2}$. A partir dos coeficientes encontrados na fórmula anterior, aplicou-se a equação a seguir para cada variável dietética:

$$
E(Y \mid Q)=\hat{v}+\hat{\lambda Q}
$$

sendo que $\hat{Y}$ refere-se a um novo valor obtido, representando o questionário de frequência al imentar calibrado. N esse sentido, $\lambda$ é o fator de calibração responsável pela nova gradação de todos os valores "brutos" do QFAA².

As médias do QFAA calibrado foram comparadas às médias do R24h, utilizando-se Teste t-Student emparelhado.

Para todas as análises estatísticas, definiu-se como nível de significância $p<0,05$.

Este estudo foi aprovado pelo Comitêde Ética em Pesquisa da Faculdade de Saúde Pública da Universidade de São Paulo. Deve ser ressaltado que só puderam participar do estudo os alunos que trouxeram o Termo de Consentimento Livre e Esclarecido preenchido eassinado pelo seu responsável. 


\section{Resultados}

Dos 74 adolescentes que perfizeram a amostra final, $71,6 \%$ eram do sexo feminino. A média de idade observada foi de 12,7 anos (desvio-padrão de 0,7 ano). Observou-se uma prevalência de excesso de peso de $37,9 \%$, sendo que a obesidade foi maisfrequente dentreas meninas ( $11,3 \%$ contra $4,8 \%$ dos meninos).

Somente foi observada diferença entre os sexos para a média de ferro do Recordatório de 24 horas; portanto, as análises de calibração foram realizadas para o grupo.

A Tabela 1 apresenta os valores dos coeficientes de correlação de Pearson entre os valores do QFAA e os valores médios dos R24h brutos,

Tabela 1. Coeficientes de Correlação de Pearson entre os dados obtidos pelo QFAA e por R24h.

\begin{tabular}{lrrr}
\hline & \multicolumn{3}{c}{$r$} \\
\cline { 2 - 4 } $\begin{array}{l}\text { Variável } \\
\text { dietética }\end{array}$ & \multicolumn{3}{c}{$\begin{array}{c}\text { Bruto } \\
\text { pjustado } \\
\text { pela energia }\end{array}$} \\
\hline Energia & $0,30^{*}$ & - & 0,44 \\
Proténa & 0,23 & 0,20 & 0,32 \\
Lipídio & $0,27^{*}$ & $0,26 *$ & 0,46 \\
Carboidrato & 0,31 & 0,12 & 0,17 \\
Fibra & $0,42^{* *}$ & $0,28^{*}$ & 0,40 \\
Colesterol & $0,35^{*} *$ & 0,17 & 0,36 \\
Cálcio & $0,37 * *$ & 0,12 & 0,20 \\
Ferro & 0,22 & $-0,05$ & $-0,07$ \\
Vitamina C & $0,39^{*}$ & $0,41^{* *}$ & 0,58 \\
Retinol & $0,25^{*}$ & $0,28^{*}$ & - \\
\hline
\end{tabular}

${ }^{*} p<0,05 ;{ }^{* *} p<0,01$

Tabela 2. Coeficientes da regressão de calibração para as variáveis dietéticas ajustadas pela energia.

\begin{tabular}{lrrrr}
\hline $\begin{array}{l}\text { Variável } \\
\text { dietética }\end{array}$ & $\boldsymbol{v}$ & \multicolumn{1}{c}{$\mathrm{IC} 95 \%$} & $\boldsymbol{\lambda}$ & \multicolumn{1}{c}{$\mathrm{IC} 95 \%$} \\
\hline Energia & 1532,45 & 984,$90 ; 2080,00$ & $0,25^{*}$ & 0,$06 ; 0,44$ \\
Proteína & 60,07 & 37,$54 ; 82,60$ & 0,22 & $-0,04 ; 0,48$ \\
Lipídio & 57,72 & 34,$21 ; 81,22$ & $0,26^{*}$ & 0,$03 ; 0,49$ \\
Carboidrato & 244,97 & 153,$01 ; 336,93$ & 0,12 & $-0,11 ; 0,36$ \\
Fibra & 12,32 & 6,$41 ; 18,23$ & $0,32^{*}$ & 0,$06 ; 0,57$ \\
Colesterol & 142,24 & 62,$47 ; 222,02$ & 0,24 & $-0,08 ; 0,55$ \\
Cálcio & 551,71 & 297,$00 ; 806,42$ & 0,18 & $-0,16 ; 0,52$ \\
Ferro & 17,22 & 12,$14 ; 22,30$ & $-0,07$ & $-0,37 ; 0,24$ \\
Vitamina C & $-2,48$ & $-36,66 ; 31,71$ & $0,40^{*}$ & 0,$19 ; 0,60$ \\
Retinol & 156,49 & 84,$77 ; 228,20$ & $0,17^{*}$ & 0,$03 ; 0,31$ \\
\hline
\end{tabular}

$v$ : intercepto da equação deregressão decalibração; $\lambda$ : coeficientedecalibração obtido com variáveis dietéticas ajustadas pela energia; ${ }^{*} p<0,05$. ajustados pela energia e os coeficientes corrigidos pela variabilidade intrapessoal. As variáveis dietéticas que apresentaram coeficientes de correlação significativos para valores brutos foram energia, lipídio, carboidrato, fibra, colesterol, cálcio, vitamina C e retinol. A pós o ajuste pela energia, os coeficientes variaram de- 0,05 a 0,41 , sendo que as correlações para carboidrato, colesterol e cálcio perderam a significância estatística. Com o ajuste dos coeficientes pela variabilidade intrapessoal, houveum incremento para a maioria das variáveis dietéticas, variando entre $-0,07$ (ferro) e 0,58 (vitamina C).

A Tabela 2 mostra os valores de coeficientes da regressão, incluindo-seo fator de calibração $\lambda$, variando de-0,07 (ferro) a 0,40 (vitamina C). As variáveisque apresentaram significância estatística para o coeficientedeinclinação da reta $\lambda$ foram energia, lipídio, fibra, vitamina C e retinol.

A Tabela 3 apresenta valores para as variáveis dietéticas ajustadas pela energia e calibradas. Foram observadas diferenças estatísticas para todas as variáveis dietéticas, exceto cálcio, ao se compararem os valores obtidos por ambos os instrumentos.

Como também esperado pela aplicação do método de regressão linear, é possível observar que, com a calibração, as médias do QFAA calibrado são mais próximas das médias obtidas pelo R24h, com redução dos valores de desviopadrão.

Não houve mudança no posicionamento dos indivíduos quando ordenados pelos valores de consumo do QFAA antes e após a calibração. Pôde-se notar em nível individual que o valor calibrado do questionário se moveu em direção ao valor médio dos dois R24h (referência).

\section{Discussão}

Este trabalho representa um dos poucos estudos conduzidos no Brasil abordando uma metodologia de calibração de dados dietéticos e foi conduzido com o objetivo de determinar fatores de correção para o QFAA que possam ser aplicados nos dados e medidas associativas a serem obtidas na coorte do estudo principal, localizada no município de Piracicaba (SP).

Yokoo et al. ${ }^{15}$ apresentam resultados de validação e calibração de consumo de mercúrio em comunidades ribeirinhas, porém não se trata de dados dietéticos obtidos por QFA, mas sim por R24h, calibrados utilizando-se como método de referência a pesagem de alimentos. 
Tabela 3. Valores de média, desvio-padrão, IC (95\%) e mediana para energia e nutrientes ajustados pela energia e calibrados, obtidos por meio de QFAA e R24h.

\begin{tabular}{|c|c|c|c|c|}
\hline \multirow[b]{2}{*}{ Variável dietética } & \multicolumn{4}{|c|}{ Geral $(n=74)$} \\
\hline & M édia & DP & IC95\% & Mediana \\
\hline \multicolumn{5}{|l|}{ Energia (Kcal) } \\
\hline QFAA & 2748,6 & 982,6 & 2520,$9 ; 2976,2$ & 2688,0 \\
\hline R24h & 2216,3 & 822,3 & 2025,$8 ; 2406,9$ & 2028,7 \\
\hline QFAA calibrado & 2216,8 & 244,7 & 2160,$2 ; 2273,5$ & 2201,8 \\
\hline \multicolumn{5}{|l|}{ Proténa (g) } \\
\hline QFAA & 86,8 & 13,8 & 83,$6 ; 90,0$ & 85,7 \\
\hline R24h & 79,1 & 15,4 & 75,$5 ; 82,6$ & 77,7 \\
\hline QFAA calibrado & 79,1 & 3,0 & 78,$4 ; 79,8$ & 78,8 \\
\hline \multicolumn{5}{|l|}{ Lipídio (g) } \\
\hline QFAA & 102,3 & 13,5 & 99,$1 ; 105,4$ & 103,4 \\
\hline R24h & 84,0 & 13,5 & 80,$9 ; 87,1$ & 84,3 \\
\hline QFAA calibrado & 84,0 & 3,5 & 83,$2 ; 84,8$ & 84,3 \\
\hline \multicolumn{5}{|l|}{ Carboidrato (g) } \\
\hline QFAA & 390,3 & 35,7 & 382,$0 ; 398,5$ & 386,9 \\
\hline $\mathrm{R} 24 \mathrm{~h}$ & 292,2 & 35,9 & 283,$9 ; 300,6$ & 293,4 \\
\hline QFAA calibrado & 292,1 & 4,3 & 291,$2 ; 293,2$ & 291,7 \\
\hline \multicolumn{5}{|l|}{ Fibra (g) } \\
\hline QFAA & 22,5 & 5,7 & 21,$2 ; 23,8$ & 22,3 \\
\hline R24h & 19,4 & 6,5 & 17,$9 ; 20,1$ & 18,8 \\
\hline QFAA calibrado & 19,4 & 1,8 & 19,$0 ; 19,8$ & 19,4 \\
\hline \multicolumn{5}{|l|}{ Colesterol (mg) } \\
\hline QFAA & 247,5 & 62,5 & 233,$0 ; 262,0$ & 235,9 \\
\hline R24h & 200,6 & 84,5 & 181,$0 ; 220,2$ & 184,4 \\
\hline QFAA calibrado & 200,7 & 14,8 & 197,$2 ; 204,1$ & 197,9 \\
\hline \multicolumn{5}{|l|}{ Cálcio (mg) } \\
\hline QFAA & 734,7 & 143,6 & 701,$4 ; 768,0$ & 727,5 \\
\hline $\mathrm{R} 24 \mathrm{~h}$ & 681,4 & 209,6 & 632,$8 ; 729,9$ & 673,4 \\
\hline QFAA calibrado & 681,8 & 25,4 & 675,$9 ; 687,6$ & 680,5 \\
\hline \multicolumn{5}{|l|}{ Ferro $(\mathrm{mg})$} \\
\hline QFAA & 16,3 & 2,9 & 15,$6 ; 16,9$ & 15,9 \\
\hline R24h & 16,2 & 3,8 & 15,$3 ; 17,0$ & 15,8 \\
\hline QFAA calibrado & 16,2 & 0,2 & 16,$1 ; 16,2$ & 16,2 \\
\hline \multicolumn{5}{|l|}{ Vitamina C (mg) } \\
\hline QFAA & 148,5 & 68,4 & 132,$7 ; 164,4$ & 147,1 \\
\hline R24h & 56,2 & 66,7 & 40,$7 ; 71,6$ & 37,0 \\
\hline QFAA calibrado & 56,2 & 27,0 & 49,$9 ; 62,5$ & 55,6 \\
\hline \multicolumn{5}{|l|}{ Retinol $(\mu \mathrm{g})$} \\
\hline QFAA & 483,3 & 205,4 & 435,$7 ; 530,9$ & 463,7 \\
\hline $\mathrm{R} 24 \mathrm{~h}$ & 239,1 & 124,6 & 210,$2 ; 268,0$ & 230,1 \\
\hline QFAA calibrado & 239,1 & 35,1 & 231,$0 ; 247,3$ & 235,8 \\
\hline
\end{tabular}

Obs.: os valores apresentados para QFAA eR24h são ajustados pela energia.

Lopes $^{16}$ apresenta resultados de calibração de um Questionário Semiquantitativo de Frequência Alimentar (QSFA) obtidos em um inquérito populacional, realizado também no Brasil. Vale ressaltar que a metodologia de calibração utilizada no referido estudo diferiu das descritas na literatura internacional, não sendo possível comparar com os resultados do presente trabalho.

A maioria dos estudos mostra inclinaçõesmenores do que 1,0. Nesta pesquisa, os valores ob- servados dos coeficientes $\lambda$ variaram de- 0,07 (ferro) a 0,40 (vitamina $C$ ), possivelmente afetados pelos fatores descritos no início deste trabalho.

Embora os estudos a seguir não tenham sido conduzidos com adolescentes, utilizaram a mesma abordagem deste trabalho e serão citados a título de ilustração.

No estudo de Kaaks et al., observa-se que 0 coeficiente de correlação para a ingestão de lipídio $(0,22)$ foi semelhanteao apresentado antes do ajuste 
pela variância, enquanto os valores para a inclinação da reta foram distintos (Kaaks et al. ${ }^{5}$ descreveram um ë=0,38 para a ingestão de lipídio repre sentada como porcentagem da energia total).

As correlações que mostraram significância estatística obtidas neste estudo (de 0,26 a 0,41 antes do ajuste pela variância intrapessoal) foram inferiores às apresentadas por Subar et al. ${ }^{17}$ $(0,4$ a 0,7$)$, quando comparados valores do QFA e de repetidos recordatórios dietéticos.

Observando-se os $\lambda$ discutidos no trabalho de $M$ acl ntyre et al. ${ }^{18}$, em que são comparados métodos propostos por Kaaks et al. ${ }^{19}$ e Beaton ${ }^{20}$, notasequea vitamina $C$ obteve o maior $\lambda(0,55$ e 0,89$)$. Dos coeficientes obtidos com o método proposto por Kaaks et al. ${ }^{19}$, observa-se que $0 \lambda$ do presente estudo para o lipídio foi maior (os referidos autores obtiveram $\lambda=0,12$ ). M acl ntyre et al. ${ }^{18}$ apresentaram IC $95 \%$ que incluiu os valores esperados de zero para a constante e 1,0 para o fator $\lambda$, indicando a ausência de viés para esse nutriente. Neste trabalho, observou-se que apenas o intercepto da vitamina $C$ também incluiu o valor zero.

Slater et al. ${ }^{21}$, ao testarem a metodologia de calibração em banco dedados secundário deadolescentes do município de São Paulo, obtiveram coeficientes $\lambda$ de 0,89 para energia, 0,41 para carboidratos, 0,22 para lipídios e 0,20 para proténas. N este estudo, $0 \lambda$ do lipídio foi maior $(0,26)$, e o da proteína $(0,22)$ foi semel hante. Ressalta-se que no estudo citado as autoras têm como objetivo descrever a proposta metodológica; portanto, os fatores de calibração estimados não foram utilizados para a correção de dados dietéticos.

As correlações observadas entre os instrumentos no estudo de $M$ acl ntyre et al. ${ }^{18}$ (os autores não citam possíveis ajustes utilizados nos dados dietéticos) variaram de 0,22 (ferro) a 0,55 (vitamina $\mathrm{C}$ ), sendo próximas aos coeficientes brutos observados nesta pesquisa. No presente trabalho, as correlações foram próximas às do estudo citado anteriormente para energia, lipídio eferro e superior para cálcio.

Em relação ao nutriente ferro, M acl ntyre et al. ${ }^{18}$ descrevem um valor negativo no IC $95 \%$ do $\lambda$ para um dos métodos comparados, assim como o resultado obtido nessa amostra de adolescentes $(\lambda=-0,07)$. H artman eFreedman ${ }^{22}$ também afirmam em seu trabalho que valores de micronutrientes apresentaram viés positivo, com exceção do ferro. $\mathrm{Na}$ amostra estudada, observou-se a inversão do sinal do coeficiente de calibração após o ajuste pela energia.

Segundo Willett ${ }^{14}$, para que se atribua uma função a um determinado nutriente na etiologia de uma doença em virtude do seu consumo, é necessário que esse nutriente permaneça associado à doença após o ajuste pela energia. Se a energia for mais bem medida do que o nutriente que está sendo investigado, o efeito do ajuste pela energia podeir à direção oposta à pretendida, até mesmo invertendo o sinal da estimativa do parâmetro23.

No presente estudo, observou-se um decréscimo nos valores das correlações para a maioria dos nutrientes após o ajuste pela energia (exceto vitamina C e retinol). Segundo $\mathrm{W}$ illett ${ }^{14}$, isto pode ser explicado quando a variabilidade do nutrienteé depen dente de erros sistemáticos de superestimação ou de subestimação.

Em relação à violação das pressuposições do método de calibração linear, Kipnis et al. ${ }^{1}$ afirmam que quando o erro de medida está correlacionado com a exposição verdadeira, o fator de calibração pode ser negativo ou maior que 1,0 em magnitude.

Devido ao fato de questionários, R24h e registros contarem com a habilidade dos indivíduos em recordar e descrever seu consumo, podem consequentemente ter algumas fontes de erro em comum, resultando em correlações positivas entre os erros de medida, especialmente quando medidas são obtidas em período muito curto de tempo. Desta forma, a variância dos níveis de ingestão predita podeser um tanto quanto superestimada quando registros ou recordatórios são utilizados como método de referência. Uma solução prática seria a coleta de medi das de referência em um intervalo de tempo mais apropriado, separadamente, não coincidindo com a aplicação do questionário. As medidas baseadas em biomarcadores são provavelmente as mais independentes, mas são disponíveis para poucos nutrientes 5 .

Assim como nos estudos de Kaaks et al. ${ }^{5}$, Kynast-Wolf et al. ${ }^{2}$, Hoffmann et al. ${ }^{24}$ e Slater et al. ${ }^{21}$, as estimativas do desvio-padrão tornam-se estreitas para os valores calibrados, notando-se o encurtamento da escala após a abordagem da calibração.

H offmann et al. ${ }^{24}$ compararam a calibração linear com um método não linear e obtiveram uma redução dos valores de desvio-padrão da ordem de até mais de $50 \%$ em relação aos valores originais na metodologia linear. Como também justificado por Slater et al. ${ }^{21}$, uma das explicações dadas por Kynast-Wolf et al. ${ }^{2}$ seria a de que especialmente os valores extremos são afetados pela correção linear devido à pressuposição delinearidade entre os valores do QFA e os de referência. 
Na maioria das situações, a regressão de calibração (método paramétrico) fornece uma meIhoria das estimativas brutas, porém as estimativas ainda podem estar fortemente enviesadas em situações em que a variância do erro é alta ${ }^{25}$.

A exemplo do descrito por Slater et al. ${ }^{21}$, observando-se os resultados, pressupõe-se pela proximidade das médias que, após a abordagem da calibração, o erro de mensuração foi minimizado. Porém, a proposta da cali bração linear não ésuficiente para mover tais valores em nível individual quando eles se apresentam distanciados dos obtidos pelo método de referência.

Para a obtenção de coeficientes $\lambda$ maiores, sugere-se a condução de novos estudos com maior tamanho amostral ou com mais aplicações do método de referência. Kaaks et al. ${ }^{26}$ defendem a ideia de que uma única aplicação do método de referência é suficiente. Porém, deve ser levado em consideração que geralmente os autores que advogam a favor de uma única aplicação do método têm a possibilidade de conduzirem grandes estudos, com grandes amostras de população, muitas vezes da ordem demilhares deindivíduos. Carroll et al. ${ }^{27}$ defendem a ideia de que pelo menos duas aplicações do método de referência são preferíveis. Não obstante a essas contradições, nesteestudo optou-se pela aplicação de dois R24h devido à possibilidade de se corrigirem as medidas pelo erro aleatório intrapessoal e se obter o consumo habitual dos nutrientes e energia. No entanto, mais aplicações do instrumento de referência ainda podem ser necessárias.

É importante ressaltar que o QFA se torna uma ferramenta útil em estudos epidemiológicos quando o seu desenvolvimento é cuidadosamente planejado, podendo garantir maior acurácia e precisão dos dados. 0 método apresenta limitações resultantes de erros na estimativa do tamanho da porção e da frequência habitual de consumo, além de listas de al imentos incompletas ou muito extensas e que levam o entrevistado à fadiga, principalmente quando se lida com um público jovem ${ }^{27,28}$.

Alguns estudos têm visado ao desenvolvimento de uma versão simplificada do QFA, de forma que a redução das listas a menores números de alimentos permita a obtenção, na mesma popu- lação, de validade e reprodutibilidade tão satisfatórias quanto as obtidas pelo questionário original ${ }^{29,30}$. Porém, apesar de sua grande importância, ainda são escassos os trabalhos realizados com este tema, especialmente envolvendo adolescentes.

Levando-se em consideração todas as questões abordadas neste trabalho e concordandose com Rosner et al. ${ }^{31}$, pode-se considerar complexo o conhecimento da extensão dos erros de medida dietéticos em epidemiologia, particularmente quando as variáveis de exposição são medidas com diferentes graus de erro. Portanto, são necessárias investigações sobre a sua estrutura, incluindo o estudo de correlações entre os erros nos valores observados e os valores verdadeiros, como também propostas por Stürmer et $a{ }^{25} \mathrm{e}$ Day et al. ${ }^{23}$.

Vale ressaltar também que a aplicação de um modelo inadequado para a correção do erro de medidae, consequentemente, as violações às pressuposições do método podem acarretar outros problemas importantes, tais como a introdução de vieses adicionais, cuja magnitude e direção geralmente são desconhecidas ${ }^{25}$.

Não érecomendada autilização do coeficiente de calibração do ferro para a correção de informação dietética para estenutriente, visto quea inversão do sinal do $\lambda$ após o ajustepela energia não é um comportamento plausível entre dois métodos deinquérito dietético quese propõem a medir uma mesma variável. Portanto, antes do uso dos coeficientes obtidos para a correção dos dados da coorte principal, é necessário ponderar os aspectos identificados e, se possível, também pesquisar outras metodologias de calibração (como as não paramétricas ou semiparamétricas) 5,25 .

Também são propostas com este trabalho: a reformulação do instrumento, particularmente no que concerne aos itens al imentares fontes de ferro; o estudo de questionários menos extensos e com mais apelos visuais para o tipo de população estudada e que possam favorecer respostas mais acuradas pela diminuição da fadiga do entrevistado durante o preenchimento; ea inclusão de outras variáveis de ajuste no modelo como uma possibilidade de se obterem coeficientes melhores. 


\section{Colaboradores}

SM Voci participou da coleta de dados, das análises estatísticas, interpretação dos resultados, redação e revisão do artigo; B Slater, das análises estatísticas, interpretação dos resultados, redação e revisão; MV Silva, da supervisão da coleta de dados, redação e revisão; D M L M archioni, da interpretação dos resultados, redação e revisão; M RDO Latorre, das análises estatísticas e da revisão do artigo.

\section{Referências}

1. Kipnis V, Carroll RJ, Freedman LS, Li L. implications of a new dietary measurement error model for estimation of relative risk: application to four calibration studies. Am J Epidemiol 1999; 150(6):642651.

2. Kynast-Wolf G, Becker N, Kroke A, Brandstetter BR, Wahrendorf J, Boeing $\mathrm{H}$. Linear regression calibration: theoretical framework and empirical results in EPIC, Germany. Ann Nutr M etab 2002; 46:2-8.

3. Michels KB, Bingham $S A$, Luben $R$, Welch $A A$, Day $N E$. The effect of correlated measurement error in multivariate models of diet. Am J Epidemiol 2004; 160(1):59-67.

4. Rosner B, Willett WC, Spiegelman D. Correction of logistic regression relative risk estimates and confidence intervals for systematic within-person measurement error. Stat Med 1989; 8:1051-1069.

5. Kaaks R, Riboli E, van Staveren W. Calibration of dietary intake measurements in prospective cohort studies. Am J Epidemiol 1995; 142(5):548-556.

6. Freedman LS, Carroll RJ, Wax Y. Estimating the relation between dietary intake obtained from a food frequency questionnaire and true average intake. Am J Epidemiol 1991; 134(3):310-321.

7. Cade J, Thompson R, Burley V, Warm D. Development, validation and utilization of food-frequency questionnaires: a review. Public H ealth Nutr 2002; 5(4):567-587.

8. World Health Organization (WHO). WHO Reference 2007: growth reference 5-19 years. [document na Internet] [acessado 2004 mar 14]. Disponível em: http://www.who.int/growthref/en/

9. Slater B, Fisberg RM, Philippi ST, Latorre M RO. Validation of a semi-quantitative adolescents food frequency questionnaire applied at a public school in São Paulo, Brazil. Eur J Clin Nutr 2003; 57:629635.

10. Dietsys - HHHQ - DietSys Analysis Software, version 4.01 [software na Internet]. Bethesda (MD): National Cancer Institute; 1999. [acessado 2004 mar 14]. Disponível em: http://appliedresearch.cancer. gov/DietSys/software.html

11. NDS. Nutrition Data System for Research [software em CD-ROM ]. Version 2005. M inneapolis: University of M innesota, NCC Food and Nutrient Database; 2005.

12. Andrade RG, Pereira RA, Sichieri, R. Consumo alimentar de adolescentes com e sem sobrepeso do município do Rio de Janeiro. Cad Saude Publica 2003; 19(5):1485-1495.

13. Willett WC, Stampfer MJ. Total energy intake: implications for epidemiological analyses. Am J Epidemiol 1986; 124:17-27.

14. Willett WC. Nutritional epidemiology. $2^{\text {a }}$ ed. New York: Oxford University Press; 1998. 
15. Yokoo EM, Valente JG, Sichieri R, Silva EC. Validation and calibration of mercury intake through selfreferred fish consumption in riverine populations in Pantanal Mato-Grossense, Brazil. Environmental Research 2001; 86:88-93.

16. Lopes ACS. Projeto Bambuí: avaliação de instrumental metodológico para uso em inquéritos nutricionais. Calibrando o Questionário Semiquantitativo deFrequência Alimentar (QSFA) em um inquérito populacional [dissertação]. Belo Horizonte: Faculdade de M edicina, Universidade Federal de M inas Gerais; 1999.

17. Subar AF, Thompson FE, Kipnis V, Midthhune D, Hurwitz P, M cNutt S, Mclntosh A, Rosenfeld S. Comparative validation of the Block, Willett, and National Cancer Institute food frequency questionnaires: the eating at America's Table Study. Am J Epidemiol 2001; 154(12):1089-1099.

18. Maclntyre UE, Venter CS, Vorster HH, Steyn HS. A combination of statistical methods for the analysis of the relative validation data of the quantitative frequency questionnaire used in the THUSA study. Public Health Nutr 2001; 4(1):45-51.

19. Kaaks R, Riboli E, Esteve J, van Kappel $A L$, van Staveren WA. Estimating the accuracy of dietary questionnaire assessments: validation in terms of structural equation models. Stat M ed 1994; 13(2):127-142.

20. Beaton GH. Interpretation of results from diet history studies. In: Kohlmeier L. The diet history method: proceedings of the $2^{\text {nd }}$ Berlin M eeting on Nutritional Epidemiology. Nishimura: Smith-Gordon; 1991. p. 15-38.

21. Slater B, Marchioni DML, Voci SM . Aplicação de regressão linear para correção de dados dietéticos. Rev Saude Publica 2007; 41(2):190-196.

22. Hartman AM, Freedman LS. Calibrating food-frequency questionnaires: a comparison of three studies [abstract]. In: IV Calibration-validation: statistical methods. Am J Clin Nutr 1997; Supl.65:1327S-1330S. Day NE, Wong MY, Bingham S, Khaw KT, Luben R,

23. Michels KB, Welch A, Wareham NJ. Correlated measurement error: implications for nutritional epidemiology. Int J Epidemiol 2004; 33(6):1373-1381.

24. Hoffmann K, Kroke A, Klipstein-Grobusch K, Boeing $\mathrm{H}$. Standardization of dietary intake measurements by nonlinear calibration using short-term reference data. Am J Epidemiol 2002; 156(9):862-870.

25. Stürmer $T$, Thürigen $D$, Spiegelman $D$, Blettner $M$, Brenner $\mathrm{H}$. The performance of methods for correcting measurement error in case-control studies. Epidemiology 2002; 13(5):507-516.
26. Kaaks R, Ferrari P, Ciampi A, Plummer M, Riboli E. Part H. Advances in the statistical evaluations and interpretation of dietary data: uses and limitations of statistical accounting for random error correlations, in the validation of dietary questionnaire assessments. Public Health Nutr 2002; 5(6A): 969-976.

27. Carrol RJ, Pee D, Freedman LS, Brown CC. Statistical design of calibration studies. Am J Clin Nutr 1997; 65(Supl.):1187S-1189S.

28. Drewnowski A. Diet image: a new perspective on the food-frequency questionnaire. Nutr Rev 2001; 59(11):370-372.

29. Chiara VL, Sichieri R. Food consumption of adolescents: a simplified questionnaire for evaluating cardiovascular risk. Arq Bras Cardiol 2001; 77(4):332-341.

30. Thompson FE, Midthune D, Subar AF, Kahle LL, Schatzkin A, Kipnis V. Performance of a short tool to assess dietary intakes of fruit and vegetables, percentage energy from fat and fibre. Public $\mathrm{H}$ ealth Nutr 2004; 7(8):1097-1106.

31. Rosner B, Spiegelman D, Willett WC. Correction of logistic regression relative risk estimates and confidence intervals for measurement error: the case of multiple covariates measured with error. Am J Epidemiol 1990; 132(4):734-743.

Artigo apresentado em 09/04/2008

Aprovado em 16/12/2008

Versão final apresentada em 06/01/2009 